\title{
Reliable Resource Allocation for Energy Constrained Mobile Grid Systems
}

\author{
Dr. S. Thenmozhi ${ }^{1}$, S. Kavithabharathi ${ }^{2}$ \\ ${ }^{1}$ Associate Professor, Department of Computer Applications, PES University, Bangalore-85 \\ ${ }^{2}$ Assistant Professor, Department of Computer Applications, Kongu Engineering College, Perundurai
}

\begin{abstract}
In this paper, we have proposed an reliable resource allocation algorithm for energy constrained mobile grid environment. In resource selection phase, nodes are chosen according to their connectivity and residual energy. The users' mobility patterns are analyzed by the predicting the nodes prevailing time in the network. In view of avoiding task failure due to fault selection of nodes grid service reliability is analysed using reliability of matchmaking process. In resource allocation phase, optimization mechanism is applied for reducing the energy consumption and improving the application utility using a utility function. This represents the total utility of mobile grid systems that considers energy, processing power and bandwidth which does not exceed the available energy budget, resource capacity, deadline, and expense budget.
\end{abstract}

Keywords: Resource allocation, Mobile grid, Computational grid, Energy efficiency, Energy constrained

\section{Introduction}

\subsection{Mobile Grid Systems}

The way of integrating computational grids and mobile ad hoc networks is called mobile ad hoc computational grids. A computational grid is defined as a software infrastructure that is used for sharing the computing resources in distributed computing devices among mobile device by enabling the innovative applications. A mobile ad hoc network is a wire-free network made up of mobile devices (Sayed Chhattan Shah 2014). It is used for providing communication without any fixed network infrastructure and provides networking and communication services to nodes within a grid. Mobile ad hoc grid allows nodes to spontaneously form an ad hoc network which facilitate autonomous interaction, forming a grid or join an existing grid dynamically contribute to the grid and being active in the services offered by other nodes in the Grid (Sayed Chhattan Shah 2014). In mobile grid, a mobile device can act both as a resource consumer and as a service provider. As a resource consumer, mobile device requests service to a grid, while, as a provider, it participates in processing service requests actively (Sayed Chhattan Shah et al 2012).

Task failure can be caused by unpredictable node mobility across the coverage area. Within the coverage area, unpredictable node mobility can cause increased communication cost. This situation causes a constrained communication environment because of the limited power, unreliable shared communication medium, available spectrum, and node mobility. This constrained environment leads to limited bandwidth, unstable connectivity issues and high latency; thus, severe network congestion can occur as a result of frequent failure and activation of links. Nodes that are present inside the grid depend on battery for power and their power is limited. Hence, the energy must be utilized properly in order to achieve extended lifetime of the nodes. The applications' battery energy limitation is the main challenge towards enabling persistent mobile grid computing (Sayed Chhattan Shah et al 2012).

\subsection{Resource Allocation for Mobile Grid Systems}

Resource allocation is the second phase of job scheduling process in grid computing system which plays an important role in determining the overall system performance that should be suitable for mobile ad hoc environment by minimizing completion time and ensuring the completion of the tasks inspite of intermittent connection caused by the mobility of nodes and lack of power (Sri Chusri Haryanti \& RiriFitri Sari 2014). In resource allocation phase, a single or one set of resources will be selected from a group of potential resources, which meet the minimum requirements for the tasks. Allocating tasks in appropriate resources can increase the system performance of resource allocation that includes minimizing the computation time. This will increase the performance and guarantee that all tasks is completed and sent back to the initial nodes safely. But failures occur as a result of resource allocation fault; hence, a resource could not return its completed tasks. The resource node may fail to access the resource management system after some period. The best resource to fulfil the task requirement has to be found in a heterogeneous environment (Sri Chusri Haryanti \& RiriFitri Sari 2014).

The characteristics such as node mobility, constrained communication environments, energy constraint, task dependencies, and absence of fixed network infrastructure has posed many challenges in the design of resource allocation scheme for mobile ad hoc computational grids. Owing to frequent failure and activation of links, communication cost can be increased by unreliable and short-term connectivity. It can also be increased by ineffective resource allocation because of multi-hop communication. Dependent tasks exchange data throughout the execution. These tasks should be allocated simultaneously or with less delay. Otherwise, one task is allocated, and waits for data from another task that is still waiting for an allocation. In this case, the allocated task cannot proceed, but it wastes the valuable resources (Sayed Chhattan Shah et al 2012). 


\section{International Journal of Science and Research (IJSR) \\ ISSN (Online): 2319-7064 \\ Index Copernicus Value (2013): 6.14 | Impact Factor (2014): 5.611}

It is necessary to consider node mobility mode to obtain good system performance. This is due to the factor that in mobile ad hoc computational grid, a node can join and leave the grid dynamically associated with mobility [2]. Faulty selections of appropriate resources results in unfinished tasks (Sayed Chhattan Shah \& Myong-Soon Park 2011).

Energy efficiency is significant for extending the mobile devices' lifetime. Hence, it is the most important design criteria for energy constrained devices. If tasks are not effectively allocated, then the energy consumption and communication cost will be increased. This can limit the node's lifetime and cause power failure. This situation will affect the task execution on a node and dependent tasks execution on the other nodes (Sayed Chhattan Shah \& Myong-Soon Park 2011). An effective and robust resource allocation scheme is needed for minimizing the communication cost between dependent tasks. The minimization of energy can extend the time up to which the mobile devices work properly without the need for recharging or substituting batteries (Li Chunlin \& Layuan Li 2010).

\subsection{Problem Identification}

Resource selection and allocation mechanism proposed by Sayed Chhattan Shah 2014 is not efficient and reliable. It is because, in resource selection process it is possible for different kinds of failure at each possible layer due to mobility, energy management etc. which is not considered. In resource allocation process, faulty resource allocation is likely happen result in uncompleted tasks. So the resource could not return its completed tasks. Due to mismatch resource, communication traffic is expanded which leads to consuming maximum energy for completing the task.

\section{Literature Review}

Li Chunlin \& Layuan Li (2010) have proposed the energy constrained resource allocation optimization algorithm to solve the utility optimization problem. Here, the problem is decomposed into two sub-problems, and the interaction between two sub-problems is controlled by using a pricing variable and modelling the prices of different resources. This can be used to allocate the appropriate grid resources in terms of application budgets, resource capacities and application completion times. In case of price-directed approach, the nodes can share the resources specify the initial set of prices for their resources to the grid user agent. To iteratively achieve an optimal solution, the grid users update their allocations using resource provider's price policy. Grid user agents identify their optimal allocation and communicate their results to the grid resource agents in each iteration. Finally, grid resource agents update their prices and inform the new prices to the user agents.

Sayed Chhattan Shah (2014) have proposed robust resource allocation scheme to allocate the interdependent tasks in a mobile ad hoc computational grid for minimizing the task completion time and energy consumed during data transmission. Resource selection algorithm selects the nodes that remain connected for a longer period by using the past records of the mobility patterns of the user. It can also predict the next probable location to become grid members for solving the assigned task. A resource allocation algorithm is proposed to allocate the interdependent tasks to nodes with minimum transmission power. This algorithm can further reduce the data transfer cost and transmission energy consumption. The relationship between data transfer time and transmission energy consumption is analysed and a power-based routing protocol is developed for minimizing the transmission energy consumption and data transfer cost.

Sayed Chhattan Shah \& Myong-Soon Park (2011) have proposed hybrid power-based resource allocation scheme. By using transmission power control mechanism, this scheme minimizes the energy consumption and communication cost among the interdependent tasks. The dependency and task types are exploited and interdependent tasks are allocated to nodes with minimum transmission power level. A power-based algorithm addresses the problem of searching a group of closest nodes when more than one interdependent task is allocated simultaneously to the closely located nodes within a grid. This problem is formulated as a k-nearest neighbour search problem. This can choose another node with maximum weight in a grid and node among the neighbour nodes for the allocation.

Sri Chusri Haryanti \& RiriFitri Sari (2014) have proposed reliability of resource allocation in mobile ad hoc grid with tasks replication, which used two approaches for avoiding tasks failure in resource selection caused by nodes mobility and task replication for considering the mobile ad hoc grid's reliability. The first proposed approach selected the specific resource nodes which willing to share computing resources for achieving a reliable resource allocation service among all nodes with corresponding mobility, resource management system and ability to properly completing the task, After resolving the task, task replication strategy is applied to consolidate the fault tolerant mechanism for enhancing the service reliability.

Sri Chusri Haryanti \& RiriFitri Sari (2014) have proposed improving resource allocation performance in mobile ad hoc grid with mobility prediction by extending energy-efficient resource allocation (EERA) for finding a cluster resource node considering the mobility of the grid members. In which the tasks allocated to resource nodes which willing to share resources in the group that have ability to communicate with the requesting node until completing its tasks then reached with minimum transmission power. After identifying group of resource nodes mobility prediction is added which obtained from the difference of received signal strength to identify nodes that have corresponding mobility with the requesting node those nodes are gathered in a cluster for completing the task.

\section{Reliable Resource Allocation}

\subsection{Overview}

In this proposal, we propose to design an reliable resource allocation algorithm for energy constrained mobile grid environment. In resource selection phase, based on the connectivity and residual energy, nodes are selected. By analyzing the past records of the user's mobility patterns,

\section{Volume 5 Issue 1, January 2016}




\section{International Journal of Science and Research (IJSR) \\ ISSN (Online): 2319-7064}

Index Copernicus Value (2013): 6.14 | Impact Factor (2014): 5.611

nodes that can be connected for a longer period are predicted (Sayed Chhattan Shah 2014 ). The nodes that have the same next probable location become grid members for solving the assigned task.

To avoid task failure due to fault selection of nodes grid service reliability is analysed using reliability of matchmaking process (Sri Chusri Haryanti \& RiriFitri Sari 2014). The process comprises of identifying and monitoring resources, eradicating failed resources, and matching the resource requests of a service with the registered resources.

In resource allocation phase, energy constrained resource allocation optimization mechanism is applied for reducing the energy consumption and improving the application utility using a utility function (Li Chunlin \& Layuan Li 2010). It represents the total utility of mobile grid systems that considers energy, processing power and bandwidth which does not exceed the available resource capacity, energy and expense budget, and deadline. Figure-1 shows the block diagram of the process.

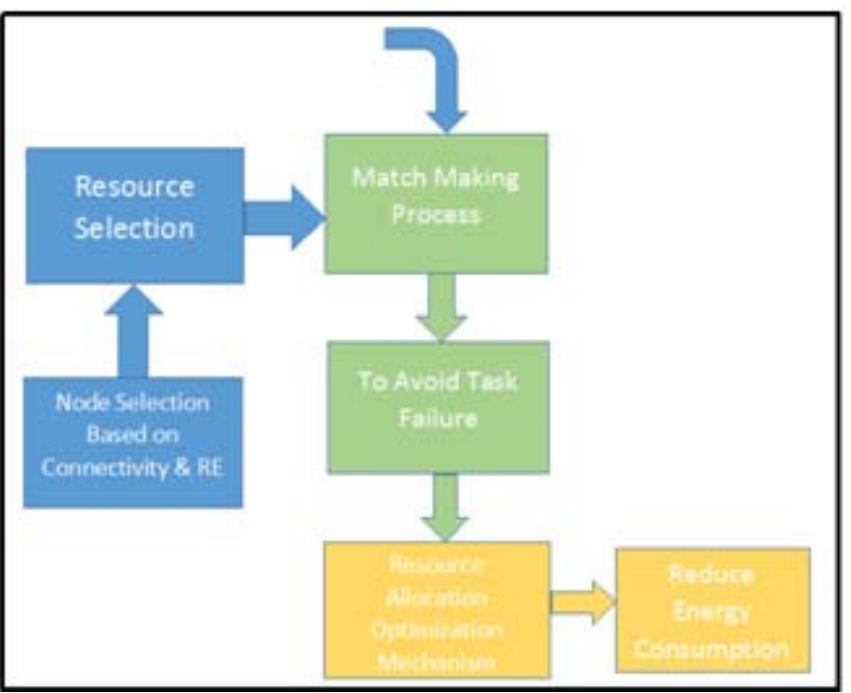

Figure 1: Block diagram

\subsection{Resource Selection}

Here in the resource selection phase, the nodes are chosen based on connectivity and residual energy.

\subsubsection{Estimation of Connectivity}

The resource selection is made in such a way, with the nodes which remains connected for a longer period of time. Initially the history of mobility pattern of a user is maintained and with the pattern next probable location is predicted. In order to solve the assigned task, the nodes that have the same next probable location can form the computational grid.

By measuring if every pair of nodes in the network is connected by either a single hop or multiple hops (reachability in graph theory), connectivity is calculated, which is given by,

$$
R=\frac{N_{-} \text {Con }}{\left(\begin{array}{c}
N \\
2
\end{array}\right)}
$$

where $\mathrm{N}$ is the number of nodes in the network N_Con is the number of connected pairs of nodes

\section{$\mathrm{R}$ is the reachability}

This method can measure only the availability of a link between two nodes in the network.

The connectivity measure is given by,

$$
C=\frac{N_{-} \text {Con }}{\left(\begin{array}{l}
N \\
2
\end{array}\right)} * \frac{\sum_{i=1}^{Q} M i}{Q}
$$

where $\mathrm{Q}$ is a subset of $\mathrm{N}$ and comprises the nodes that are actually (physically) connected. $\mathrm{M}_{\mathrm{i}}$ is the metric for the link $i$.

\subsubsection{Residual Energy}

The nodes in the resource selection phase are selected by means of Residual Energy. The residual energy can be calculated as

$$
E_{R}=E_{T}-E_{C}
$$

where $E_{T}-$ Total energy,

$E_{C}$ - Energy consumed.

\subsection{Matchmaking Process}

On the way to evade task failure due to fault selection of nodes grid service reliability is evaluated using Reliability of Matchmaking Process. This process is used to detect and monitor the resources, eliminate the failed resources, and compare the resource requests of a service with the registered resources.

During the matchmaking process, some requests may be mismatched with the wrong resource. This can cause matchmaking failure. The assumptions for matchmaking failure are given as follows,

- Let $\beta(\mathrm{k})$ be the failure rate of matchmaking process, which is a function to $\mathrm{k}$ number of faults.

- If matchmaking failure occurs, the program will notify the resource management system (RMS) to remove the faults.

- Removing the fault may sometimes introduce some other new faults. The occurrence of a new generating fault follows a Poisson distribution. The rate of new faults is constant (v).

Using the continuous time Markov chain (CTMC) matchmaking reliability is modelled. The model is represented in the below figure.

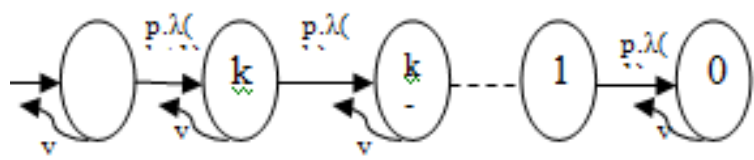

Figure 2: Continuous Time Markov chain (CTMC) matchmaking reliability model

Matchmaking time for a request is much smaller than the entire life of the resource allocation service. The failure rate can be calculated by,

$$
\beta=\frac{\eta_{f}}{\eta_{t}^{\bar{\tau}}}
$$

where $\eta_{f}$ is the number of mismatched requests 


\section{International Journal of Science and Research (IJSR)}

$\eta_{t}$ is the number of requests

$\bar{\tau}$ is the expected time to complete each requests.

The probability that all requests are correctly matched to their required resources with the given $\mathrm{X}$ requests is given by

$$
\begin{array}{r}
R_{\text {matc } h}=\exp (-\lambda \cdot X \cdot \bar{\tau}) \\
\mathrm{a}=\exp \left(\frac{-\eta_{f}}{\eta_{t}} X\right) \\
i=\exp \left(\lambda_{m} \lambda\right)
\end{array}
$$

\subsection{Resource Allocation Optimization}

We are applying the energy constrained resource allocation optimization mechanism in the resource allocation phase for reducing the energy consumption and improving the application utility using a utility function.

\subsubsection{Representation of Utility Function}

The utility function represents the total utility of mobile grid systems that considers energy, processing power and bandwidth which does not exceed the available resource capacity, deadline, energy budget, and expense budget. Grid users can update their allocations according to the resource provider's price policy and approach an optimal solution iteratively.

The grid user agents identify their optimal allocation individually and communicate their results to the grid resource agents for each iteration. For each node in the system, the energy consumption rate is measured by Joules per unit time.

Let $e_{i}^{n}$ denotes energy dissipation due to grid application $i^{\prime} \mathrm{s}$ nth job, $t_{i}^{n}$ denotes execution time of job $\mathrm{n}$ of grid application $i$ on the grid node, and er denotes energy consumption rate of energy resource 1 .

The energy dissipation of grid application $i$ 's nth job is given below as,

$$
e_{i}^{n}=e r \cdot t_{i}^{n}
$$

For each grid application $A_{i}$, the energy consumed by all grid jobs of $A_{i}$ must satisfy the following condition,

$$
\sum_{n=1}^{N} e_{i}^{n} \leq e_{i}^{l}
$$

At this moment, energy constrained resource allocation optimization problem in mobile grid is defined as a constraint optimization problem. The utility of the system $\left(\mathrm{U}_{\text {system }}\right)$ is defined as the sum of grid application utilities. $\mathrm{U}_{\text {system }}$ is given by

$$
U_{\text {system }}=\sum_{i=1}^{I} U_{i}\left(e_{i}^{l}, x_{i}^{j}, y_{i}^{k}\right)
$$

In mobile grid, the energy constrained resource allocation optimization can be formulated by:

$$
\operatorname{Max} U_{\text {system }}=\sum_{i=1}^{I} U_{i}\left(e_{i}^{l}, x_{i}^{j}, y_{i}^{k}\right)
$$

where $e_{i}^{l}$ denotes energy obtained by grid application $i$ from energy 1.

$x_{i}^{j}$ denotes CPU allocation obtained by grid application $i$ from the computing resource provider $\mathrm{j}$

$y_{i}^{k}$ denotes bandwidth allocation obtained by grid application $i$ from the network resource provider $\mathrm{k}$.

\subsection{Overall Algorithm}

1) At first for the resource selection nodes are chosen with the help of connectivity and residual energy.

2) Initially the history of mobility pattern of a user is maintained and with the pattern next probable location is predicted.

3) Next to this we are using a reliability match making process, in order to avoid the task failure due to the selection of fault nodes.

4) The process identifies new resources, examines the resources, eliminates the failed resources, and matching the resource requests of a service to the registered resources.

5) Finally we are using the utility function in the energy constrained resource allocation optimization mechanism for minimizing the energy consumption and to improve the application of the process.

\section{Simulation Results}

\subsection{Simulation Setup}

In this section, we analyse the performance of energy efficient reliable resource allocation algorithm by an extensive simulation study with the help of network simulator (NS-2) [10]. The simulation topology is illustrated in Figure 2. We compare our results with energy efficient robust allocation (EERA) technique [4]. Various simulation parameters are provided in Table 1.

Table 1: Simulation Parameters

\begin{tabular}{|c|c|}
\hline No. of Nodes & 18 \\
\hline Area Size & 1000 X 1000 \\
\hline Mac & 802.11 \\
\hline Simulation Time & $20 \mathrm{sec}$ \\
\hline Traffic Source & CBR \\
\hline Packet Size & $250,500,750$ and 1000 \\
\hline Initial Energy & $10.1 \mathrm{~J}$ \\
\hline Rate & $200,210,220,230,240$ and $250 \mathrm{~kb}$ \\
\hline Propagation Model & TwoRayGround \\
\hline Antenna Type & OmniAntenna \\
\hline
\end{tabular}

\subsection{Performance Metrics}

The performance is evaluated based on the following metrics.

Average Execution Delay: It measures the average delay occurred while executing a given task. 


\section{International Journal of Science and Research (IJSR) \\ ISSN (Online): 2319-7064 \\ Index Copernicus Value (2013): 6.14 | Impact Factor (2014): 5.611}

Average Success Ratio: It is the ratio of the number of tasks executed successfully and the total number of tasks submitted.

Average Energy: It is the average energy consumption of all nodes in executing the tasks.

Drop: It is the number of packets dropped during the data transmission.

\subsection{Results}

\section{A. Varying the number of tasks}

The number of tasks to be executed is varied from 10 to 50 with task size of 250 bytes.

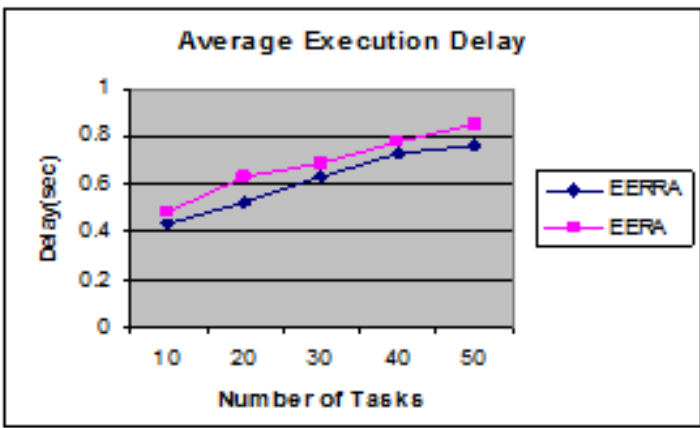

Figure 3: Execution Delay for Varying Tasks

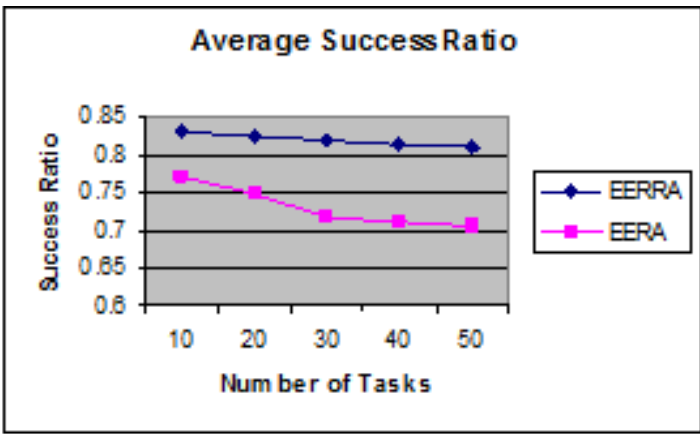

Figure 4: Success Ratio for Varying Tasks

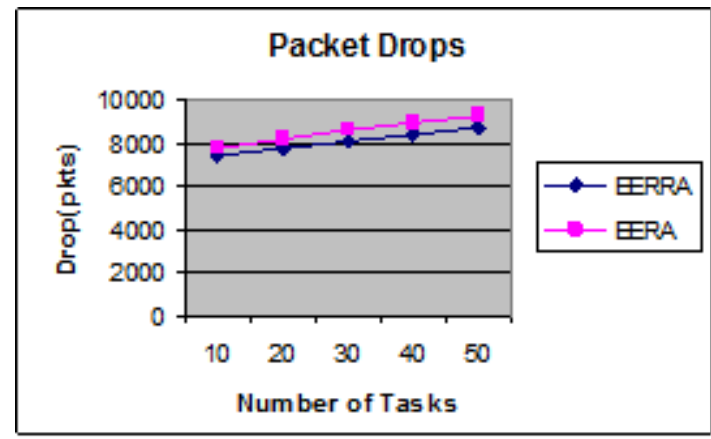

Figure 5: Packet Drops for Varying Tasks

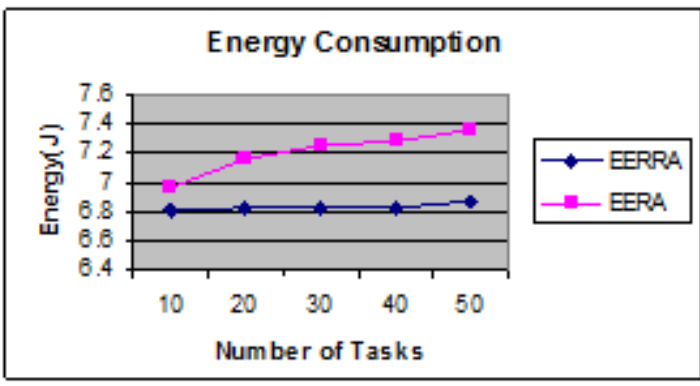

Figure 6: Energy Consumption for Varying Tasks
Figures 3 to 6 show the results of execution delay, success ratio, packet drop and energy consumption for varying number of tasks. When comparing EERRA and EERA, we infer that EERRA outperforms ERRA by $10 \%$ in terms of delay, $11 \%$ in terms of success ratio, $6 \%$ in terms of packet drop and 5\% in terms of energy consumption

\section{B. Based on Packet Size}

In the second experiment, the packet size is varied as 250 , 500,750 and 1000 bytes with rate $250 \mathrm{~kb}$.

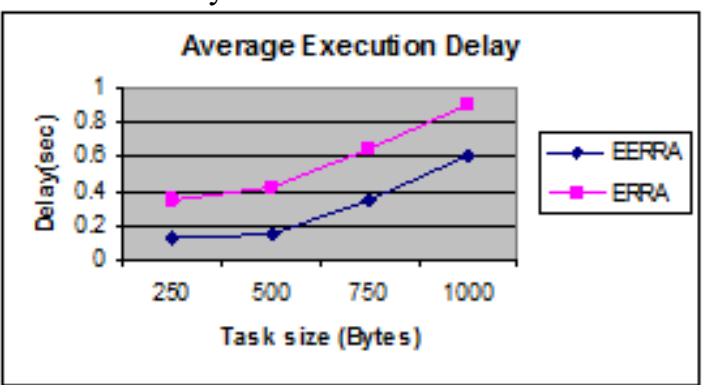

Figure 7: Execution Delay for Varying Task size

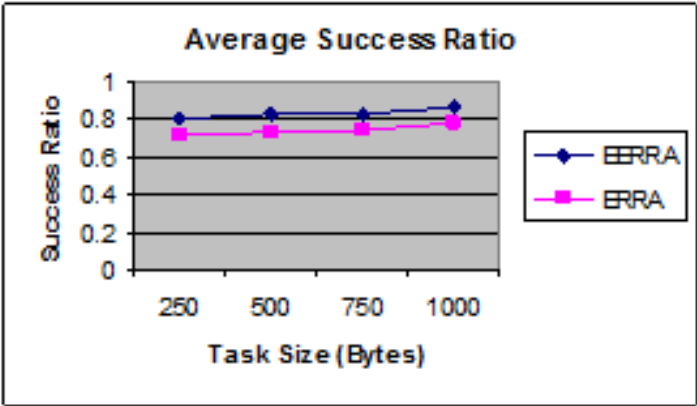

Figure 8: Success Ratio for Varying Task size

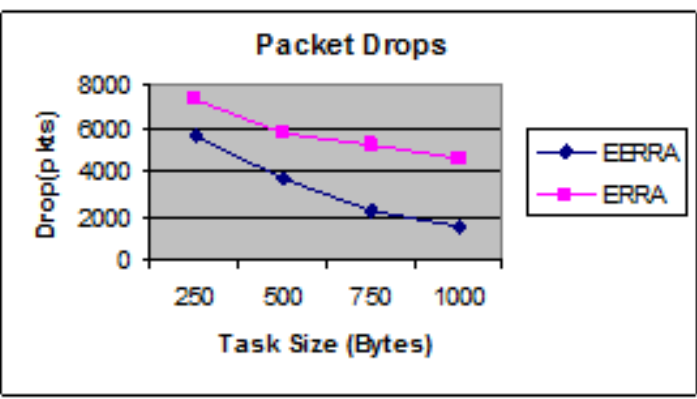

Figure 9: Packet Drops for Varying Task size

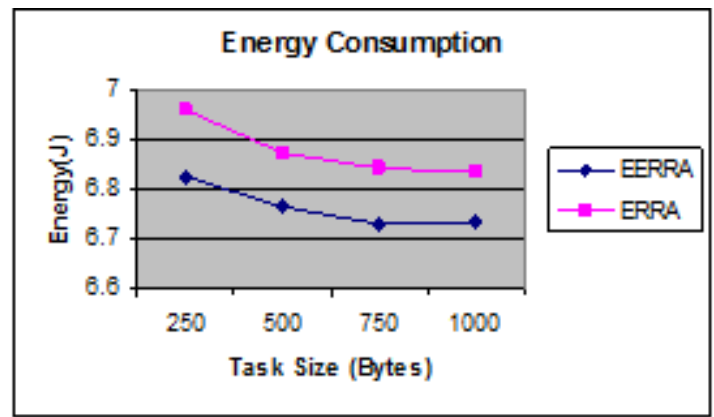

Figure 10: Energy Consumption for Varying Task size

Figures 7 to 10 show the results of execution delay, success ratio, packet drop and energy consumption for varying task size. When comparing EERRA and EERA, we infer that EERRA outperforms ERRA by $52 \%$ in terms of delay, $10 \%$ 


\section{International Journal of Science and Research (IJSR) \\ ISSN (Online): 2319-7064}

Index Copernicus Value (2013): 6.14 | Impact Factor (2014): 5.611

in terms of success ratio, $45 \%$ in terms of packet drop and $2 \%$ in terms of energy consumption. on Intelligent Green Building and Smart Grid (IGBSG), IEEE, pp.1-4.

\section{Conclusion}

Here in this proposal we have designed a reliable resource allocation algorithm for energy constrained mobile grid environment. In the resource selection phase, nodes are chosen according to connectivity and residual energy. Nodes that are connected for a longer period are predicted by analyzing the past information of mobility patterns of the user. For eliminating the task failure due to fault selection of nodes grid service reliability is analysed using Reliability of Matchmaking Process. In resource allocation phase, energy constrained resource allocation optimization mechanism is applied for reducing the energy consumption and improving the application utility using a utility function. This represents the total utility of mobile grid systems that considers energy, processing power and bandwidth which does not exceed the available resource capacity, energy and expense budget, and deadline.

\section{References}

[1] Li, Chunlin \& Layuan Li 2010, 'Energy efficient resource management in mobile grid', Mobile Information Systems, vol. 6, No. 2, pp.193-211.

[2] Li Chunlin \& Li Layuan 2010, 'Energy constrained resource allocation optimization for mobile grids', Journal of Parallel and Distributed Computing, vol. 70, No. 3, pp. 245-258.

[3] Samar Sajadian, Alia Ibrahim, Edison Pignaton de Freitas \& Tony Larsson 2011, 'Improving Connectivity of Nodes in Mobile WSN', Proceedings of International Conference on Advanced Information Networking and Applications.

[4] Sayed Chhattan Shah \& Myong-Soon Park 2011, 'Energy Efficient Resource Allocation in Mobile Ad Hoc Computational Grids', IEEE Proceedings of International Conference on Communication Systems and Networks (COMSNETS), pp. 1-10.

[5] Sayed ChhattanShaha, Qurat-Ul-Ain Nizamanib, Sajjad Hussain Chauhdaryc \& Myong-Soon Park 2012, 'An effective and robust two-phase resource allocation scheme for interdependent tasks in mobile ad hoc computational Grids', Journal of Parallel and Distributed Computing, vol. 72, No. 12, pp. 1664- 1679.

[6] Sayed Chhattan Shah 2014, 'Energy efficient and robust allocation of interdependent tasks on mobile ad hoc computational grid', Concurrency and Computation: Practice and Experience.

[7] Sri Chusri Haryanti \& RiriFitri Sari 2011, 'Effect of RPGM and Gauss Markov Node Mobility for Resource Allocation Performance in Mobile Ad Hoc Computational Grid', International Journal of Computer Science and Network Security(IJCSNS), vol.11 No.6.

[8] Sri Chusri Haryanti \& RiriFitri Sari 2014, 'Reliability of Resource Allocation in Mobile Ad Hoc Grid with Tasks Replication', Journal of Computers, vol. 9, No. 2.

[9] Sri Chusri Haryanti \& RiriFitri Sari 2014, 'Improving Resource Allocation Performance in Mobile Ad Hoc Grid with Mobility Prediction', International Conference 